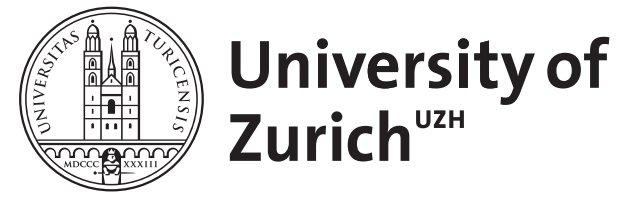

Zurich Open Repository and Archive

University of Zurich

Main Library

Strickhofstrasse 39

CH-8057 Zurich

www.zora.uzh.ch

Year: 2012

\title{
Monoklonale Gammopathie unklarer Signifikanz (MGUS) und Multiples Myelom
}

Schleiffenbaum, Boris

DOI: https://doi.org/10.1024/1661-8157/a001137

Posted at the Zurich Open Repository and Archive, University of Zurich ZORA URL: https://doi.org/10.5167/uzh-71689

Journal Article

Accepted Version

Originally published at:

Schleiffenbaum, Boris (2012). Monoklonale Gammopathie unklarer Signifikanz (MGUS) und Multiples Myelom. Praxis, 101:1521-1531.

DOI: https://doi.org/10.1024/1661-8157/a001137 


\section{Monoklonale Gammopathie unklarer Signifikanz (MGUS) und Multiples Myelom}

MGUS und Multiples Myelom sind klonale Plasmazelldyskrasien.Sie stellen die beiden Enden im Kontinuum einer malignen Plasmazell-Infiltration des Knochenmarks dar, wobei das MGUS durch eine geringe Plasmazellinfiltration $(<10 \%)$ und fehlende klinische Komplikation definiert ist und im Gegensatz dazu das Multiple Myelom durch eine hohe Plasmazellinfiltration (>10\%), bzw. durch den Nachweis einer Sekundärfolge der Plasmazelldyskrasie. Die ein Multiples Myelom definierenden Sekundärkomplikation kann man sich mit Hilfe des Wortes «CRAB» merken: Erhöhte Kalziumwerte (hyperçalcemia, Ca>0,25 mmol// oberhalb der Normgrenze oder $>2,75 \mathrm{mmol} /$ )), Niereninsufffizienz (renal insufficiency; Kreatinin >173 $\mu \mathrm{mol} / \mathrm{l}$ ), Anämie (Hb-Abfall >20 g/l unter die Normgrenze oder $\mathrm{Hb}<100 \mathrm{~g} / \mathrm{l}$ ),

Knochenfrakturen bzw. Osteolysen ( $\underline{b} o n e$ fractures, radiologisch). Zudem besteht vor allem in den späteren Phasen der Myelom-Erkrankung eine erhöhte Infektneigung (>2 bakterielle Infekte in 12 Monaten). Hyperviskositätssyndrom, Polyneuropathie und AL-Amyloidose sind Komplikationen der Paraproteinämie die sowohl beim MGUS als auch beim Multiplen Myelom auftreten können und damit unabhängig von der Plasmazellinfiltration.

Sowohl MGUS wie auch dem Multiplen Myelom liegt eine klonale Proliferation von Plasmazellen zugrunde. Diese terminal differenzierten Effektorzellen der B-Lymphoyzten-Reihe produzieren physiologischerweise Antikörper (Immunglobuline) als Antwort auf den Antigen-Kontakt im Rahmen vor allem von Infektionen und sind somit der zelluläre Eckstein der plasmatischen Immunabwehr.

Das initiale molekulare Ereignis in der Entstehung des malignen Klons ist nicht bekannt. Wohl aber weiss man, dass die Erkrankung gehäuft auftritt nach Strahlen- oder Benzen-Exposition. Die bei den Plasmazelldyskrasien nachweisbaren zytogenetischen Aberrationen spielen sicher in der Progression der Erkrankung eine Rolle, der genaue Mechanimus ist jedoch auch hier unbekannt.

Das bunte klinische Bild, das von vollkommener Beschwerdefreiheit bis hin zu schwersten invalidisierenden Schmerzen reicht, ergibt sich einerseits aus dieser unterschiedlichen Progression, andererseits aus dem Isotyp und der Menge des sezernierten, nicht-funktionellen Immunglobulins (Paraprotein) und der so aufgrund von negativen FeedbackMechanismen ausgelösten Hypogammaglobulinämie. Diese ist Ursache der Infektanfälligkeit der Myelom-Patienten. An dieser Stelle sollte angemerkt werden, dass Paraproteinämien auch im Rahmen von Non-Hodgkin-Lymphomen gefunden werden können, eine Paraproteinämie damit nicht gleichbedeutend ist mit der Diagnose Plasmazelldyskrasie (dies gilt insbesodere für den Isotyp IgM: Lymphoplasmozytisches Lymphom/M. Waldenström). Das Paraprotein kann, muss aber nicht, über den tubulären Ausfall und die Toxizität der nierengängigen Leichtketten zur Niereninsuffizienz führen, ebenso wie zur Polyneuropathie, oder kann als Amyloid im Gewebe abgelagert werden. Auch die Interaktion der malignen Plasmazellen mit ihrem Mikroenvironment im Knochenmark ist ganz unterschiedlich: Es kann, muss aber nicht, über eine Interaktion mit den ortsständigen Osteoklasten zum Knochenabbbau und damit zur Hyperkalziämie, zu Osteolysen und im Extremfall zu pathologischen Frakturen kommen. Die von Patient zu Patient unterschiedliche Interaktion mit der Erythropoiese (Hepcidin) wiederum bestimmt das Ausmass der die Plasmazelldyskrasie begleitenden Anämie. Erst im Endstadium der Erkrankung hat die Knochenmarksinfiltration direkte pathophysiologische Auswirkungen und es kommt zur Knochenmarksverdrängung und -ineffizienz (Neutropenie, Thrombopenie, verstärkte Anämie).

Die Erkrankung tritt im mittleren und höheren Alter auf (nur 35\% $<65$ Jahre!) und betrifft Frauen etwas häufiger als Männer (M:F=1,4:1). 
Die Prognose des MGUS ist im Grunde gut, die des Multiplen Myeloms eher schlecht. Eine MGUS-Phase geht bei so gut wie allen Patienten der Diagnose eines Multiplen Myeloms voraus. Nicht ein jedes MGUS geht jedoch in ein Multiples Myelom über (durchschnittlich 1\%/Jahr, siehe auch Tab. 1). Für das MGUS und das Multiple Myelom gibt es inzwischen relativ gut definierte Subgruppen, die sehr unterschiedlich rasch voranschreiten und im Falle des Multiplen Myeloms ganz unterschiedlich auf die Therapie ansprechen (siehe unten).

\section{((hier etwa Tab. 1))}

\section{Im Artikel verwendete Abkürzungen:}

$\begin{array}{ll}\text { FISH } & \text { Fluoreszenz-in-situ-Hybridisierung } \\ \mathrm{Hb} & \text { Hämoglobin } \\ \text { MGUS } & \text { Monoklonale Gammopathie unklarer Signifikanz } \\ \text { MRD } & \text { Minimal residual disease } \\ \mathrm{NHL} & \text { Non-Hodgkin-Lymphom } \\ \text { OS } & \text { Gesamtüberleben (overall survial) } \\ \text { PFS } & \text { Progression free survival } \\ \text { PR } & \text { Partial response } \\ \text { sCR } & \text { Stringent complete remission } \\ \text { sFLC } & \text { Serum-Freie-Leichtketten (serum free light chains) } \\ \text { TTP } & \text { Time to progression, in diesem Fall zum symptomatischen Multiplen Myelom } \\ \text { VGPR } & \text { Very good partial response" }\end{array}$

\section{Klinik ((Ü2))}

Sowohl MGUS als auch ein Multiples Myelom kann sich mit den Symptomen und Zeichen einer Polyneuropathie (meist sensorisch distal betont, Hyp- und Parästhesie, Ausfall der Muskeleigenreflexe) oder einer Amyloidose (Herz- oder Niereninsuffizienz, Polyneuopathie, gastrointestinale Symptomatik) präsentieren.

Die Klinik des MGUS ist sonst defintionsgemäss stumm, während sich das Multiple Myelom vor allem das Beschwerdebild seiner Komplikationen zeigt (siehe unten).

Fortgeschrittene Stadien eines Multiplen Myeloms, mit rezidivierenden Infekten als Zeichen der Immundefizienz oder gar Allgemeinsymptomen (Schwäche, Müdigkeit, Fieber, Gewichtsverlust, Nachtschweiss) oder Folgen einer Zytopenie (wiederum Infekte bei Neutropenie, bzw. hämorrhagische Diathese in Folge einer Thrombopathie bzw. Thrombasthenie) sieht man heute bei Erstdiagnose selten.

\section{Komplikationen ((Ü2))}

Abgesehen von Polyneuropathie und Amyloidose (siehe oben) zeigt das MGUS keine Komplikationen. Spontanfrakturen der langen Röhrenknochen, der Rippen vor allem aber des Achsenskeletts (Rückenschmerzen! Höhenminderung), die gerne im Rahmen einer generaliserten Osteoporose gedeutet werden, müssen immer auch an ein Multiples Myelom denken lassen.

Seltener präsentiert sich ein Multiples Myelom heutzutage mit dem Beschwerdebild eines manifesten Nierenversagens (Müdigkeit, Pruritus, Foetor), einer schweren Anämie (Müdigkeit, Dyspnoe und sogar Angina pectoris und Synkopen) 
oder einer Hyperkalziämie (psychiatrische oder zentralnervöse Symptomatik; Diabetes insipidus; Erbrechen, Obstipation). Im Rahmen der Abklärung einer Niereninsuffizienz bzw. einer Anämie, die bei asymptomatischen oder interkurrent anderweitig erkrankten Patienten als Zufallsbefunde diagnostiziert wurden, sollte der Arzt jedoch relativ bald auch an die Diagnose eines Multiplen Myeloms denken und entsprechende Abklärungsschritte einleiten (siehe unten).

\section{Abklärungsstrategie ((Ü1))}

In der Anamnese sollte nach Knochenschmerzen, einer Verminderung der Körpergrösse, rezidivierende Infekte und Müdigkeit als Ausdruck einer Niereninsuffizienz oder auch einer Anämie gefragt werden. Dyspnoe, Angina pectoris oder Synkopen können auf eine schwere Anämie hinweisen, während die Atemnot auch Ausdruck einer Kardiomyopathie im Rahmen einer Amyloidose sein kann, die sich im übrigen auch mit gastrointestinalen Symptomen

(Schluckbeschwerden, Obstipation/Diarrhoe) oder wie die Leichtketten-Erkrankung oder die Paraprotein-assoziierte tubuläre Nephritis mit den Zeichen einer Niereninsuffzienz bzw. eines nephrotischen Syndroms (Ödeme) präsentieren kann. Zentralnervöse Störungen oder Persöhnlichkeitsveränderungen hingegen können auf eine Hyperkalziämie hinweisen.

In der körperlichen Untersuchung sind pathologische Frakturen der langen Röhrenknochen zum Teil sehr einfach zu diagnostizeren. Rippenfrakturen aber können z.B. sehr lange unentdeckt bleiben, während Einbruchfrakturen der Wirbelkörper klinisch oft nicht bemerkt werden (Gibbus; lokale Klopfdolenz). Die klinischen Zeichen der Anämie und der Niereninsuffizienz sind relativ unspezifisch. Trotzdem sollte der internistische Status ebenso wie die persönliche und die Systemanamnese unbedingt vollständig sein, um einerseits einen Infekt, und andererseits Begleiterkrankungen zu erfassen, die in der Altersgruppe der Myelom-Patienten häufig sind.

Eine erste Blutuntersuchung sollte ein grosses Blutbild mit morphologischer Differenzierung (Anämie; Hinweise auf die Genese der Anämie; Rouleaux-Formation und Proteinfilm als Hinweise auf eine Paraproteinämie; sehr selten bei Diagnose: Plasmazell-Leukämie), das Kreatinin (Niereninsuffizienz), ein CRP (Infekt), das Kalzium (Hyperkalzämie) und einen Urinstatus (nephrotisches Syndrom; cave: die Stix-Untersuchung erfasst eine Bence-Jones-Proteinurie nicht!) umfassen.

Dreh- und Angelpunkt in der Diagnostik des MGUS und des Multiplen Myeloms ist für den Hausarzt und Praktiker die Serumeiweisselektrophorese, ob sie nun im Rahmen einer allgemeinen Abklärung oder auch spezifisch mit dem Verdacht auf eine Plasmazelldyskrsasie durchgeführt wurde: Zeigt sich hier ein M-Gradient, so muss die klonale Genese dieses «peaks» in der Elektrophorese mit Hilfe der Immunfixation bewiesen und der Isotyp des Paraproteins klassifiziert werden. Die Immunfixation hat bei der Diagnostik unter dem Verdacht auf ein Multiples Myelom eine besondere Bedeutung, weist sie doch eine etwa 10 fache Sensitivität verglichen mit der Serumeiweisselektropherese auf. Im Hinblick auf die Formen des Multiplen Myeloms, die vornehmlich Leichtketten sezernieren (20-25\%! «Leichtkettenerkrankung») ist die Bestimmung der «freien Leichtketten» im Serum ein Muss bei klinischem Verdacht auf ein Multiples Myelom (alternativ: Bence-Jones-Proteine im Urin).

Bei Vorliegen einer Paraproteinämie (inkl. klonal vermehrter freier Leichtketten im Serum) kann bzw. sollte in Abhängigkeit von der Höhe des Paraproteins und der Klinik (Tab. 1) eine Knochenmarkspunktion erfolgen: Das Knochenmarkaspirat (EDTA/Ausstrichpräparate; eine relativ schnelle Diagnostik) ermöglicht dabei die morphologische Beurteilung (Plasmoblastom?), die Immunphänotypisierung (wichtig in morphologisch schwierigen Grenzfällen in der Abgrenzung zum Immunblastom und zum Plasmazell-reichen Lymphomplasmozyoidem NHL) und die Karyotypisierung, 
inkl. FISH (sehr wichtig in der Prognostik, siehe unten), während die Biopsie (inkl. Immunhistologie) oft erst die definitive Diagnose, insbesondere aber die Beurteilung des Infiltrationsgrades des Knochenmarks erlaubt. Bei klinischem Verdacht auf ein Multiples Myelom ist eine Knochenmarkspunktion immer indiziert, auch bei fehlender Paraproteinämie oder fehlender Erhöhung der sFLC («nicht-sezernierendes» Multiples Myelom, 2-3\%). Abbildung 1 zeigt die typische Infiltration des Knochenmarks durch asynchrone, grössenpolymorphe, Plasmazellen, die z.T. auch zwei- und mehrkernig sein können und deren Klonalität in der Immunhistologie bewiesen werden kann (Abb. 2).

Unabhängig von Röntgenaufnahmen bei Frakturverdacht sollte mit Hilfe von Radiographien des Schädels, der langen Röhrenknochen, der Rippen und des Achsenskelettes nach Osteolysen gesucht werden und Multiples Myelom ausgeschlossen werden. Heutztage ist hier die Computertomographie im Knochenfenster eine valable Alternative.

((hier etwa Abb. 1und 2))

Diagnosekriterien und Differenzialdiagnose ((Ü1))

\section{Diagnosekriterien ((Ü2))}

Ein MGUS diagnostiziert man, wenn eine Paraproteinämie/erhöhte sFLC vorliegen und ein Multiples Myelom bzw. eine andersartige B-Zellproliferation/NHL (siehe oben) ausgeschlossen werden konnte (siehe unten, Tab. 1). Somit kann diese Diagnose letztendlich mit Sicherheit nur nach erfolgter Knochenmarkspunktion gestellt werden.

Ein Multiples Myelom wird diagnostiziert, wenn >10\% klonale Plasmazellen im Knochenmark nachgewiesen werden können, bzw. wenn Komplikationen im Sinne der CRAB-Kriterien vorliegen (siehe oben; Tab. 1). Schlussendlich wird noch zwischen einem «smouldering myeloma» mit erhöhtem Anteil klonaler Plasmazellen im Knochenmark und einem klinisch manifesten, und damit therapiebedürftigem Multiplen Myelom mit CRAB-Komplikationen unterschieden (cave: aufgrund einer Polyneuropathie oder einer Amyloidose kann auch einmal ein «smouldering myeloma» oder selbst ein MGUS therapiebedürftig sein).

Um als Kriterium für ein therapiebedürftiges Myelom zu gelten, muss die Nierensuffizienz nicht nur ein bestimmtes

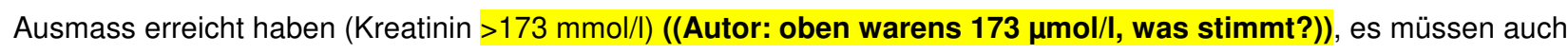
andere Ursachen ausgeschlossen worden bzw. unwahrscheinlich sein (arterielle Hypertonie, Diabetes mellitus, Stauungsnieren, Glomerulonephritis und andere). Ähnliches gilt natürlich auch für die anderen Kriterien, insbesondere die Anämie ( $\mathrm{Hb}<100 \mathrm{~g} / \mathrm{l})$. Zusätzlich zu Kreatinin (renale Anämie) und CRP («anemia of chronic disease»), sollten hier Ferritin/GPT (Eisenmangel), Vitamin B12, Folat und TSH (Hypothrese) bestimmt werden. Auch die sensorische, distal betonte Polyneuropathie hat andere, viel häufigere Ursachen als eine Paraproteinämie, bzw. ein Multiples Myelom (vor allem Alkohol, Diabetes mellitus).

\section{Differenzialdiagnose ((Ü2))}

Neben der Plasmazelldyskrasie kann auch eine klonale B-Zell Proliferation/NHL Ursache einer Paraproteinämie sein. Dies gilt insbesondere für den IgM Subtyp, der meist im Rahmen eines lymphoplasmozytoiden NHL gesehen wird. Die Unterscheidung ist von grösster Wichtigkeit, da die und Therapie dieses NHL ganz anders ist als die von MGUS und Multiplem Myelom.

Auch andere Malignome treten mit zunehmendem Alter gehäuft auf und das MGUS ist im Alter fast schon endemisch (>70 Jahre: ca. 10\%). Somit sollte bei Osteolysen trotz Vorliegen einer Paraproteinämie und Nachweis klonaler 
Plasmazellen im Knochenmark immer auch die Möglichkeit eines Zweittumors erwogen werden. Bei hohem Verdacht (bekanntes Mamma- oder Prostata-Karzinom) ist hier u.U. sogar die direkte Punktion des osteolytischen Herdes selbst eine diagnostische Möglichkeit.

\section{[1.}

\section{Prognose ((Ü2))}

Die Prognose des MGUS wird bestimmt durch den Isotyp des Paraproteins, die Höhe des Paraproteins und der sFLC (Tab. 1). Das Intervall der Kontrolluntersuchung und die Notwendigkeit einer Knochenmarksuntersuchung wird somit auch durch die Risikogruppe bestimmt, in die der jeweilige Patient fällt (durchschnittliche Progression MGUS zu einem Multiples Myelom 1\%/Jahr; Tab. 1). Es empfiehlt sich im Allgemeinen ein Kontrollintervall von 1 Jahr, für Patienten mit hohem Risiko von sechs Monaten, um so den Übergang in ein Multiples Myelom frühzeitig zu diagnostizieren und schwerwiegende Komplikationen zu verhindern. Ein steiler Anstieg der $\mathrm{k} / \lambda$-Ratio scheint am Ende der MGUS-Phase dem klinisch manifesten Mylom vorauszugehen (Leichtketten-MGUS vor Leichtketten-Myelom!).

Als wichtigstes Kriterium in der Prognostik des Multiplen Myeloms hat sich ein den letzten Jahren der Karyotyp der malignen Plasmazellen etabliert. Eine Hochrisikogruppe wird dabei durch Patienten mit 17p Deletionen und t14;16 und t14;20 Translokalisationen definiert, ein mittleres Risiko haben die Patienten mit hypoploidem Chromosomensatz und Translokalisation t4;14 sowie Deletion 13 q, während alle Patienten mit einem normalen Karyotyp oder einer Hyperploidie zur Niedrig-Risko-Gruppe gezählt werden können.

Als Zusatzkriterien für die Prognose des Multiplen Myeloms und damit auch die Wahl einer spezifischen Therapie gelten

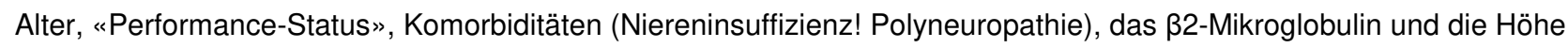
des Albuminspiegels bzw. der LDH sowie der ISS-Score (I: $\beta 2-$ Mikroglobulin <3,5 mg/l, Albumin >3,5 mg/l; II: nicht I oder III; III: $\beta 2-M i k r o g l o b u l i n ~>5,5$ mg/l). Auch das Ansprechen auf die Therapie («minimal residual disease» MRD) ist von Bedeutung für die Prognose der Erkrankung.

\section{Therapie ((Ü2))}

Die Notwendikeit zur Therapie ergibt sich aus dem Nachweis, z.T. auch der Schwere von Komplikationen (s.o.). Die Therapie selbst gehört in die Hand des spezialisierten Hämatologen oder Onkologen. Nach erfolgter Induktions-, Transplantations- und Konsoldationstherapie können die Verlaufskontrollen aber durchaus in Zusammenarbeit von Spezialist und Hausarzt erfolgen, wobei sich die Kontrollintervalle nach dem individuellen Patienten richten sollten (Klinik; Serumeiweisselektropherese, Immunfixation, sFLC; Ca, Kreatinin; Blutbild; Urinstatus).

Die Art und die Strategie der Therapie werden durch das Alter des Patienten, selten auch durch die Prognose bei Diagnosestellung und immer auch durch die Art der zu erwartenden Nebenwirkungen einer geplanten Chemotherapie bestimmt.

Als Standardtherapiestrategie für jüngere Patienten (<65 Jahre als arbiträre Grenze der Studienprotokolle; je nach Gesundheitszustand ist die Tranplantation jedoch sicher und durchführbar auch für Patienten im Alter 70-75 Jahre) gilt heute ein Schema, das eine Induktionsphase mit anschliessender autologer Stammzelltransplantation (Single oder Tandem-Transplantation; Tandem-Transplantation am ehesten für Hoch-Risiko Patienten bzw. bei ungenügendem Ansprechen), eine daran anschliessende Konsolidationsphase und/oder eine Maintenance-Phase umfasst. Die allogene Transplantation ist in der Therapie des Multiplen Mymeloms weiterhin sehr umstritten. Je nach Prognose und Verlauf des individuellen Falles und der Verfügbarkeit eines Familien- oder Fremdspenders wird man aber unter Umständen 
auch eine allogene Transplantation in Erwägung ziehen (zytogenetische Hoch-Risiko-Gruppe, siehe unten; schlechtes Ansprechen auf die Induktionstherapie).

Bei älteren Patienten (>65 Jahre, bzw. >75 Jahre, s. o.), für die eine Stammzelltransplantation nicht mehr in Frage kommt, schliesst sich die Konsolidationsphase direkt an die Induktionsphase an.

Als Standardtherapie, vor allem für niereninsuffiziente und Hoch-Risiko-Patienten, in der Induktionsphase gilt heute das VMP-Schema (Velcade®/Bortezomib $1,3 \mathrm{mg} / \mathrm{m}^{2}, \mathrm{~d} 1,4,8,11$, Alkeran®/Melphalan $9 \mathrm{mg} / \mathrm{m}^{2}$, d1-4, jeden 2. Zyklus, Prednison $60 \mathrm{mg} / \mathrm{m}^{2}$, jeden 2. Zyklus, d1-4; Zyklus 21d, 8 Zyklen). Dieses Therapie ist der konventionellen Behandlung mit Alkylantien und Steroiden nicht nur in der Remissionrate und der Tiefe der Remission, sondern auch im PFS und Gesamtüberleben (OS) überlegen. Unter dem Schema kann es zu Zytopenien kommen, Prednison hat die für Kortikosteroide typischen Nebenwirkungen (verschlechterte Glukosetoleranz, erhöhter Blutdruck, Infektneigung), eigentliche cushingoide Bilder sind aber eher selten, und Velcade® kann relativ schwere, schmerzhafte Polyneuropathien auslösen oder auch einen Herpes zoster reaktivieren.

Alternativ stehen heute zudem die Immunmodulatoren Thalidomid (NW: Müdigkeit, Polyneuropathie, Thrombembolien) und Revlimid®/Levalidomid (NW: Zytopenien; geringe Polyneuropathie, Thrombembolien, mit Aspirin beherrscht; unter Umständen vermehrt Sekundärkarzinome; schlechter geeignet in der Niereninsuffizienz) zur Verfügung. Neue Proteasom-Inhibitoren (Typ Velcade®/Bortezomib) bzw. Immunmodulatoren wie Carfilzomib (36 mg/m² iv, d1, 2, 8, 9, 16; 28d Zyklus) und Pomalidomide (4 mg/d po, d1-21, 28 d Zyklus) sowie Myelom-spezische Antikörper (antiCSI, Elotuzumab, 10/20 mg/kgKG iv, d1, 8, 15, 21; 28d Zyklus; anti-CD56, Lorovotuzumab, 75 mg/m², d1, 8, 15; 28d Zyklus) sind in der klinischen Erprobungsphase. In manchen Therapie-Schemata wird statt Alkeran®/Melphalan ein anderes Alkylans (Cyclophosphamide, liposomales Doxorubicin, Bendamustin) verwendet. Prednison wird in manchen Schemata durch Dexamethason ersetzt.

In der Konsolidationsphase finden wiederum Velcade®/Bortezumib, Alkeran®/Melphalan und Prednison bzw. Dexamethason bzw. alternativ Thalidomid®/Revlimid® Verwendung. Ziel ist die Vertiefung des therapeutischen Erfolges, so z.B. von einem partial response (PR) zu einem very good partial response (VGPR), bis hin zur stringent complete remission, (sCR).

Die Wertigkeit der Maintenance-Therapie mit Velcade®/Bortezomib $\left(1,3 \mathrm{mg} / \mathrm{m}^{2}, \mathrm{~d} 1,4,8,11\right.$ alle drei Monate) oder Thalidomid (50 mg/d p.o., Dauertherapie; nicht bei Hochrisiko-Patienten) bzw. Revlimid® (10 mg/d 21/28d; nicht bei Hochrisiko-Patienten) wirkt wahrscheinlich vor allem günstig bei Patienten mit relativ guter Prognose (Thalidomid®, Revlimid()). Sie ist auch heute noch umstritten, insbesondere, da bei einem Rezidiv die Salvage-Therapie zum Teil mit den gleichen schon in der Induktions- und Konsolidationsphase verwendeten Medikamenten erfolgreich ist. Für Revlimid® wurde zudem eine Vermehrung der Sekundärkarzinome diskutiert.

Ältere Patienten (>70 Jahre) können und sollen prinzipiell genauso behandelt werden wie jüngere Patienten, bei denen eine Stammzelltranplantation nicht mehr infrage kommt. Der zu erwartende therapeutische Vorteil muss aber noch sorgfältiger gegen die zu erwartenden, im Alter oft schwereren Nebenwirkungen der Therapie (Polyneuropathie) abgewogen werden, insbesondere wenn schwerwiegendere Begleiterkrankungen bekannt sind.

Leider muss abschliessend gesagt werden dass trotz grossen in den letzten Jahren erzielten Fortschritten in der Therapie des Multiplen Myeloms und einer damit deutlich verbesserten Morbidität und Mortalität der Erkrankung auch heute eine Heilung des Multiplen Myeloms selten, wenn überhaupt, zu erwarten und möglich ist. 


\section{Fallbericht zu MGUS und Multiples Myelom}

\begin{tabular}{|c|c|}
\hline & Kommentar ((Ü1)) \\
\hline $\begin{array}{l}\text { Anlässlich einer Routine-Untersuchung wird bei } \\
\text { dem damals 75-jährigen Patienten eine } \\
\text { Panzytopenie festgestellt. Der Patient ist } \\
\text { beschwerdefrei, insbesondere bestehen keine } \\
\text { Allgemeinsymptome wie Müdigkeit, } \\
\text { Gewichtsverlust, Nachtschweiss oder Fieber. Er } \\
\text { ist in regelmässiger hausärztlicher Kontrolle } \\
\text { wegen eines Typ-2-Diabetes mellitus und einer } \\
\text { arteriellen Hypertonie bei Adipositas und } \\
\text { metabolischem Syndrom. Es liegt jedoch schon } \\
\text { eine hypertensive Kardiomyopathie vor. Wegen } \\
\text { eines intermittierendem Vorhofflimmerfns wurde } \\
\text { er zweimal elektroablatiert. Zudem ist eine leichte } \\
\text { Niereninsuffizienz bekannt. } \\
\text { Die Zuweisung erfolgt zur weiteren Abklärung der } \\
\text { Panzytopenie. Der Hausarzt äussert den } \\
\text { Verdacht auf ein myelodysplastisches Syndrom. }\end{array}$ & $\begin{array}{l}\text { Angesichts einer relativ flächendeckend } \\
\text { engen ärztlichen Überwachung wird } \\
\text { heute eine Anämie häufig erfasst bevor } \\
\text { sie symptomatisch ist. } \\
\text { Die leichte Niereninsuffizienz, die man in } \\
\text { der Folge der arteriellen Hypertonie und } \\
\text { des Diabetes interpretierte, hätte wohl } \\
\text { eine geringgradige Anämie erklären } \\
\text { können, nicht aber eine Panzytopenie. } \\
\text { Die gleichzeitige Verminderung des Hb, } \\
\text { der Thrombozyten und der Neutrophilen } \\
\text { Granulozyten hatten den Hausarzt den } \\
\text { Verdacht auf eine genuine } \\
\text { Knochenmarkserkrankung äussern } \\
\text { lassen. }\end{array}$ \\
\hline 1 Befunde des Patienten ((Ü1)) & Kommentar ((Ü1)) \\
\hline $\begin{array}{l}\text { Status ((Ü2)) } \\
\text { Afebril, } 35,5^{\circ} \mathrm{C} \text {, guter AZ, leichte Stamm- } \\
\text { Adipositas (BMI 25,0). } \\
\text { Puls 60/min, unregelmässig, >10 ES/min; es fehlt } \\
\text { der Puls der linken A. dorsalis pedis; Blutdruck } \\
165 / 100 \text { mmHg, kardiopulmonal kompensiert. } \\
\text { Auch der abdominelle Befund ist unauffällig, } \\
\text { insbesondere keine Organomegalie. } \\
\text { Unauffälliger Neurostatus. }\end{array}$ & $\begin{array}{l}\text { Der erhöhte Blutdruck ist u.U. durch die } \\
\text { Situation einer Erstuntersuchung erklärt. } \\
\text { Der unregelmässige Puls hingegen weist } \\
\text { wohl auf eine rhythmologische Instabilität } \\
\text { auch nach den zwei stattgehabten } \\
\text { Elektroablationen. } \\
\text { Ein unauffälliger Untersuchungsbefund, } \\
\text { insbesondere bei vermehrtem Bauchfett, } \\
\text { schliesst eine Splenomegalie nicht aus, } \\
\text { die in Zusammenhang mit einer } \\
\text { Thrombopenie bzw. Neutropenie, wie sie } \\
\text { bei diesem Patienten besteht, immer } \\
\text { gesucht bzw. ausgeschlossen werden } \\
\text { muss. }\end{array}$ \\
\hline Aktuelle Therapie ((Ü2)) & Kommentar ((Ü1)) \\
\hline $\begin{array}{l}\text { Meto-Zerok® }{ }^{\circledR} \times 25 \text { mg, Lisinopril 1x5 mg, } \\
\text { Marcoumar® n. V. }\end{array}$ & $\begin{array}{l}\text { Es besteht eine antihypertensive } \\
\text { Therapie und eine Prophylaxe bei } \\
\text { intermittierendem Vorhofflimmern. Der } \\
\text { Diabetes mellitus wird diätetisch } \\
\text { kontrolliert. }\end{array}$ \\
\hline
\end{tabular}




\begin{tabular}{|c|c|}
\hline RoutineLabor ((Ü2)) & \\
\hline 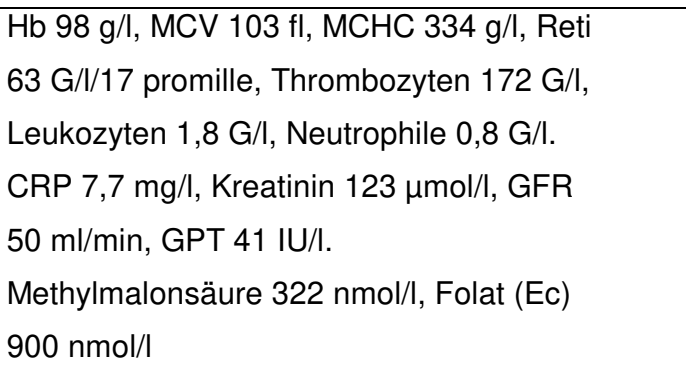 & $\begin{array}{l}\text { Die leichte Niereinsuffizienz reicht nicht, } \\
\text { um das Ausmass der makrozytären, } \\
\text { normochromen hyporegenerativen } \\
\text { Anämie zu erklären. Ein Vitaminmangel } \\
\text { besteht nicht (cave: Niereninsuffzien = } \\
\text { leicht erhöhte Methylmalonsäurewerte). }\end{array}$ \\
\hline \multicolumn{2}{|l|}{ Weitere Befunde ((Ü2)) } \\
\hline $\begin{array}{l}\text { Die Sonographie zeigt eine Splenomegalie } \\
(16 \mathrm{~cm}) \text { und eine Lebersteatose, aber keine } \\
\text { Zeichen eines zirrhotischen Umbaus bzw. einer } \\
\text { portalen Hypertension. }\end{array}$ & $\begin{array}{l}\text { Mit einem Hypersplenismus könnte man } \\
\text { nun die Neutropenie und die } \\
\text { Thrombopenie gut erklären, wobei die } \\
\text { Werte typischerweise, wie in diesem } \\
\text { Falle, schwanken. } \\
\text { Damit ändert sich die diagnostische } \\
\text { Herausforderung nun gänzlich: } \\
\text { Wir suchen nicht mehr nach einer } \\
\text { Ursache für eine Panzytopenie, sondern } \\
\text { nach einer Ursache für die } \\
\text { Splenomegalie und nach einer Ursache } \\
\text { für die Anämie, wobei es natürlich ein } \\
\text { und dieselbe Ursache sein kann. }\end{array}$ \\
\hline \multicolumn{2}{|l|}{ Erweitertes Labor ((Ü2)) } \\
\hline Ferritin 295 mg/l, TSH 1,37 mU/l; & $\begin{array}{l}\text { Ein Eisenmangel oder eine Hypothyreose } \\
\text { (als Ursache einer makrozytären Anämie) } \\
\text { liegen nicht vor. }\end{array}$ \\
\hline $\begin{array}{l}\text { Die Elektrophorese zeit einen M-Gradienten von } \\
7,5 \mathrm{~g} / \mathrm{l}, \text { Paraprotein IgG kappa } 12,83 \mathrm{~g} / \mathrm{l}(6,64- \\
\text { 14,94); IgA 0,68 g/l }(0,57-3,24), \mathrm{lgM} 0,21 \mathrm{~g} / \mathrm{l} \\
(0,39-1,57) ; \mathrm{sFLC} \text { kappa } 20,90 \mathrm{mg} / \mathrm{l}(3,30- \\
\text { 19,40), lambda } 3,78 \mathrm{mg} / \mathrm{l}(5,70-26,30), \mathrm{k} / \mathrm{\lambda} 5,53 \\
(0,26-1,65)\end{array}$ & $\begin{array}{l}\text { Die Paraproteinämie wie auch der } \\
\text { pathologische Quotient der freien } \\
\text { Leichtketten beweisen die } \\
\text { Plasmazelldyskrasie. } \\
\text { Die grenzwertig tiefen Werte des IgAs } \\
\text { und des IgMs weisen darauf hin, dass } \\
\text { trotz der relativ niedrigen Spiegel des } \\
\text { Paraproteins und der kappa-Leichtkette } \\
\text { im Serum die Krankheit schon } \\
\text { fortgeschritten sein könnte (Suppression } \\
\text { der normalen Plasmazellen durch den } \\
\text { malignen Klon). }\end{array}$ \\
\hline $\begin{array}{l}\text { Aspirat und Biopsie zeigen eine Infiltration des } \\
\text { Knochenmarks durch asynchrone, klonale } \\
\text { Plasmazellen (kappa; 30-40\%); die Kongorot- } \\
\text { Färbung ist negativ }\end{array}$ & $\begin{array}{l}\text { Damit ist die Diagnose eines Multiplen } \\
\text { Myeloms gestellt. Eine Amyloidose liegt } \\
\text { nicht vor. }\end{array}$ \\
\hline $\begin{array}{l}\text { Kalzium 2,23 mmol/l; Erythropietin 68,8 IU/l } \\
\text { Ein Ganzkörper-CT im Knochenfenster zeigt } \\
\text { keine Osteolysen }\end{array}$ & $\begin{array}{l}\text { Es besteht keine Hyperkalziämie und es } \\
\text { finden sich keine Hinweise auf } \\
\text { Osteolysen. }\end{array}$ \\
\hline
\end{tabular}




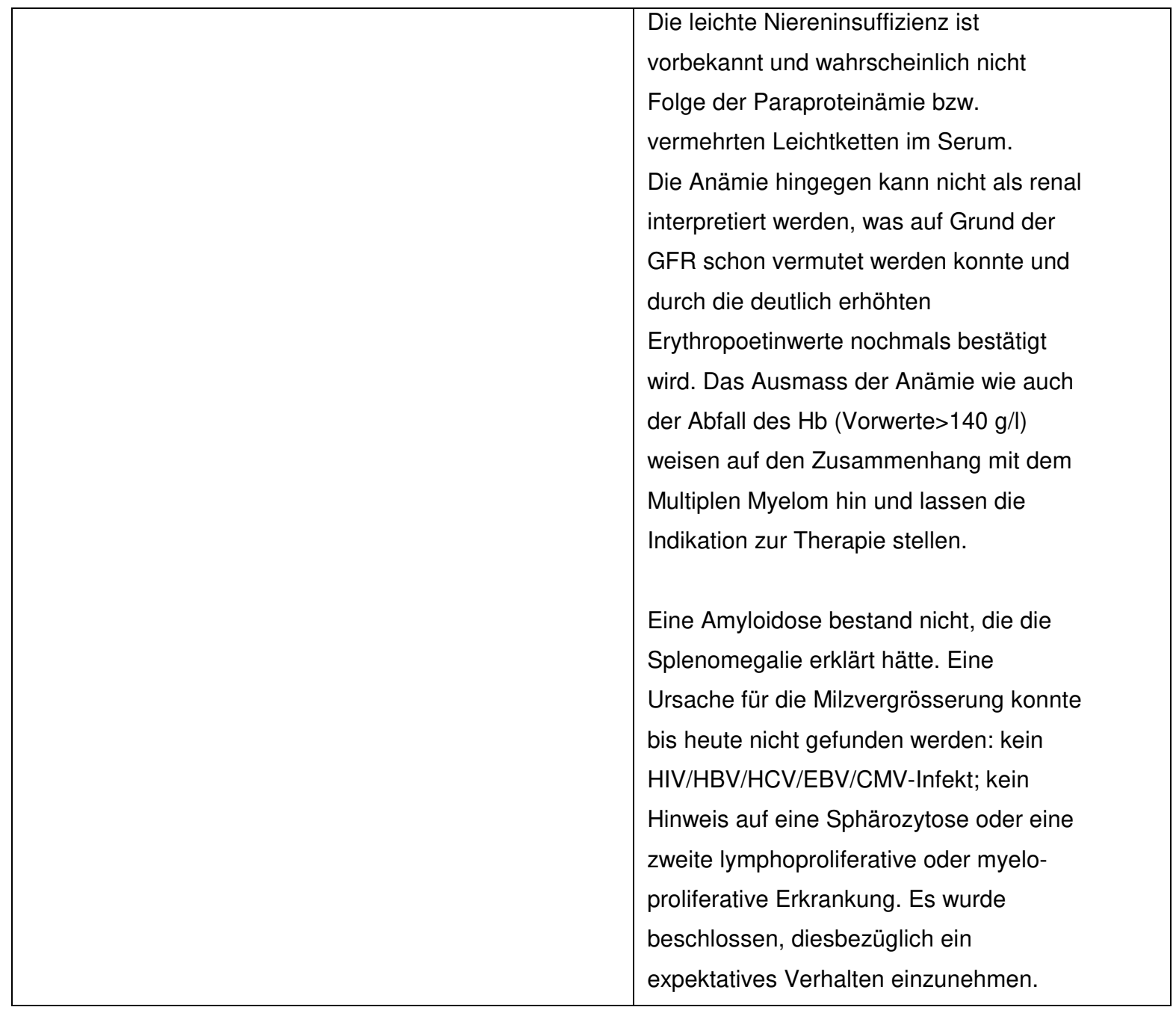

\begin{tabular}{|l|}
\hline Therapie \\
\hline Trotz des erhöhten Risikos für eine Polyneuropathie (Diabetes mellitus), entschlossen wir uns zu \\
einer Therapie mit Bortezomib (Velcade®), Melphalan (Alkeran®) und Prednison. Nach anfänglichem \\
Erfolg (Anstieg des $\mathrm{Hb}$; der niedrige Paraprotein-Spiegel bzw. sFLC kappa-Spiegel konnten nicht als \\
Verlaufsparameter dienen) zeigte sich aber eine Resistenz und die Therapie musste nach nur vier \\
Zyklen abgesetzt werden. Der Patient befindet sich jetzt unter einer Therapie mit Lenalidomid \\
(Revlimid®) und Dexamethason. Er ist wohlauf mit Hb-Werten um 105-110 g/l. Es besteht eine \\
leichtgradige Polyneuropathie.
\end{tabular}




\section{Autor}

PD Dr. med. Boris Eugen Schleiffenbaum, Hirslanden Klinik Im Park, Bellariastrasse 38, 8038 Zürich

Email: boris.schleiffenbaum@hirslanden.ch

\section{Bibliographie ((Autor: Ref. bitte im Text mit eckigen Klammern [..] erwähnen))}

1. International Myeloma Working Group: Criteria for the classification of monoclonal gammopathies, multiple myeloma and related disorders: a report of the International Myeloma Working Group. Br J Haematol 2003; 121: 749-757.

2. Ludwig H, Durie BG, McCarthy P, Palumbo A, San Miguel J, Barlogie B, et al.: IMWG consensus on maintenance therapy in multiple myeloma. Blood 2012; 119: 3003-3015.

3. Weiss BM, Abadie J, Verma P, Howard RS, Kuehl WM: A monoclonal gammopathy precedes multiple myeloma in most patients. Blood 2009; 113: 5418-5422.

4. Cavo M, Rajkumar SV, Palumbo A, Moreau P, Orlowski R, Bladé J, et al.: IMWG consensus approach to the treatment of multiple myeloma patients who are candidates for autologous stem cell transplantation. Blood 2011; 117: 6063-6073.

5. Kumar S, Giralt S, Stadtmauer EA, Harousseau JL, Palumbo A, Bensinger W, et al.: Mobilization in myeloma revisited: IMWG consensus perspectives on stem cell collection following initial therapy with thalidomide-, lenalidomide-, or bortezomib-containing regimens. Blood 2009, 114: 1729-1735.

6. Armeson KE, Hill EG, Costa LJ: Tandem autologous vs autologous plus reduced intensity allogeneic transplantation in the upfront management of multiple myeloma: meta-analysis of trials with biological assignment. Bone Marrow Transplant 2012 sep 10; [Epub ahead of print]. 


\section{Fragen zu MGUS und Multiples Myelom}

\section{Frage 1}

Eine 57-jährige Patientin mit bekannter Osteoporose präsentiert sich mit akuter Lumbago. Ein Röntgenbild zeigt eine frische LWK2-Fraktur. Die BSR ist nicht erhöht, $\mathrm{Hb}$ und Kreatinin wie auch die Transaminasen sind normal. Die Elektrophorese zeigt keinen M-Gradienten. Welche der folgenen Aussagen ist korrekt? (Einfachauswahl, 1 richtige Antwort)

a) Die vorbekannte Osteoporose erklärt das Bild ausreichend. Weitere Abklärung sind daher nicht indiziert.

b) Die normale Elektrophorese schliesst die Diagnose eines Multiplen Myeloms aus.

c) Als ergänzende Untersuchung sollte man hier noch eine Bence-Jones-Proteinurie mit dem Uro-Stix suchen.

d) Bei begründetem klinischen Verdacht kann nur eine Knochenmarksuntersuchung ein Multiples Myelom beweisen oder ausschliessen.

\section{Frage 2}

Bei einem 67-jährigen Patienten wurde ein M-Gradient von $37 \mathrm{~g} / \mathrm{l}$ festgestellt. Welche der folgenen Aussagen ist korrekt? (Einfachauswahl, 1 richtige Antwort)

a) Bei der Knochenmarkspunktion sollte neben Material für die morphologische Untersuchungen (Zytologie/Histologie) auch Material für molekulargenetische Analysen (PCR) entnommen werden, da nur aufgrund dieser Untersuchungen bei einem Multiplen Myelom die Risikostratifikation und damit die Planung der richtigen Thrapie erfolgen kann.

b) Der Paraproteinspiegel ist so hoch, dass damit die Diagnose eines Multiplen Myeloms als gesichert gelten darf.

c) Wenn keine konkomitierenden Krankheiten bestehen, sollte die Therapieplanung dieses Patienten nach einer Induktionsphase die autologe Stammzelltransplantation vorsehen.

d) Angesichts der Höhe der Paraproteinämie und des damit weit fortgeschrittenen Stadiums der Erkrankung, wird die Behandlung des Multiplen Myeloms dieses Patienten eine allogene Stammzelltransplantation einschliessen müssen.

\section{Frage 3}

Welche der folgenden Aussagen zur modernen Therapie des Multiplen Myeloms ist korrekt? (Einfachauswahl, 1 richtige Antwort)

a) Moderne Therapeutika wie Velcade® (Bortezomib) und Revlimid® (Lenalidomid) sind zwar deutlich besser verträglich als die bisherige Therapie mit Alkylanzien und Kortikosteroiden, haben aber bisher das Gesamtüberleben der Patienten nicht verlängern können.

b) Unter den neuen Therapeutika, wie z.B. Revlimid® (Lenalidomid), treten keine schwerwiegenden hämatologischen Komplikationen (wie z.B. eine Neutropenie) mehr auf.

c) Patienten unter Revlimid® (Lenalidomid) und Thalidomid müssen wegen des Thromboembolie-Risikos oral antikoaguliert werden.

d) Trotz des Risikos einer schmerzhaften Polyeuropathie gilt die Kombination Velcade® (Bortezomib), Alkeran ${ }^{\circledR}$ (Melphalan), Prednison heute als Standard-Induktionstherapie des Multiplen Myeloms.

\section{Frage 4}

Bei einem 78-jährigen, bisher gesunden Patienten, der sich wegen Rückenschmerzen beim Hausarzt gemeldet hatte, wurde die Diagnose eines Multiplen Myeloms gestellt (Knochenmarksinfiltrationsgrad 70\%). Welche der folgenden Aussagen ist korrekt? (Einfachauswahl, 1 richtige Antwort)

a) Angesichts des Alters des Patienten verbietet sich eine Therapie mit Revlimid® (Lenalidomid). 
b) Die Therapie mit Velcade® (Bortezomib), Alkeran® (Melphalan) und Prednison kann ambulant erfolgen. Der Patient sollte aber engmaschig ärztlich überwacht werden, um mögliche Komplikationen frühzeitig erkennen und behandeln zu können.

c) Sonst gesunde, ältere Patienten vertragen die Therapie mit Velcade® (Bortezomib), Alkeran® (Melphalan) und Prednison nicht und sollten daher konventionell induziert werden.

d) Die autologe Stammzelltransplantation ist die Methode der Wahl, da sie durch die Anwendung der neuen Therapeutika wie Velcade ${ }^{\circledR}$ (Bortezomib) und Revlimid® (Lenalidomid) auch für ältere Patienten gut verträglich geworden ist.

\section{Frage 5}

Welche der folgenen Aussagen zurm MGUS ist nicht korrekt? (Einfachauswahl, 1 richtige Antwort)

a) Das MGUS ist prinzipiell nicht behandlungsbedürftig.

b) Das MGUS ist prinzipiell nicht behandlungsbedürftig. Eine Polyneuropathie kann aber eine Indikation zur Therapie darstellen.

c) Patienten mit einem MGUS sollten jährlich ärztlich nachkontrolliert werden (Klinik, Blutbild, Elektrophorese, Immunfixation, sFLC; Kalzium).

d) Das MGUS geht so gut wie immer einem Multiplen Myelom voraus (durchschnittliche Rate 10\%/Jahr). 
DOI 10.1024/1661-8157/a001138

((PX 25_Rubrik CME-Antworten))

\section{Fragen zu MGUS und Multiples Myelom aus PRAXIS Nr. 24}

\section{Frage 1}

Antwort d) ist richtig.

Die vorbekannte Osteoporose könnte die Fraktur zwar auch erklären. Eine Osteoporose schliesst ein Multiples Myelom aber nicht aus, ebensowenig wie eine normale Elektrophorese, da in einem Teil der Fälle das Paraprotein nur durch die sensitivere Methode der Immunfixation erkannt wird, bzw. wenn ein Leichtketten-Myelom oder ein nicht-sezernierendes Myelom vorliegt. Der Nachweis von Bence-Jones Proteinen im Urin dient zwar der Diagnose des Leichtketten-Myeloms, der Uro-stix ist methodisch hierfür aber nicht geeignet.

Nur die Knochenmarkspunktion kann ein Multiples Myelom beweisen oder ausschliessen.

\section{Frage 2}

\section{Antwort c) ist richtig.}

Die Diagnose eines Multiplen Myeloms muss immer zytologisch, besser noch histologisch erfolgen. PCR-Methoden spielen heute in der Diagnostik und Prognostik des Multiplen Meloms (im Gegensatz zur Zytogenetik) noch keine Rolle. Bei einem einem sonst gesunden Patenten kann auch im Alter von 67 Jahren noch eine autologe Stammzelltransplantation Teil der Therapie des Patienten mit Multiplem Myelom sein, da so mit der höchsten Wahrscheinlichkeit die tiefsten Remissionen erzielt werden können. Die allogene Stammzelltransplantation bietet wahrscheinlich auch beim jüngeren Patienten im Allgemeinen keine Vorteile gegenüber der autologen Transplantation und sollte beim älteren Patienten nicht durchgeführt werden.

\section{Frage 3}

Antwort d) ist richtig.

Auch das Gesamtüberleben der Patienten einem Multiplen Myelom wird durch die neuen Therapeutika wie Velcade® (Bortezomib) und Revlimid® (Lenalidomid) verlängert. Die Standard-Therapie des Multiplen Myeloms ist heute deswegen Velcade $\AA$ in der Kombination mit Alkeran $\AA$ (Melphalan) und Prednison. Aber auch die modernen Therapeutika, obwohl insgesamt sicher sehr gut verträglich, haben Nebenwirkungen: So kann es unter Revlimid® durchaus zu schweren Neutropenien kommen, und sowohl Thalidomid wie auch Revlimid® erhöhen das Thrombembolie-Risiko. Als Gegenmassnahme genügt hier jedoch die Gabe von Aspirin® (1x100 mg/d), eine orale Antikoagulation ist nicht notwendig.

\section{Frage 4}

Antwort b) ist richtig.

Auch für ältere Patienten ist die Therapie mit Velcade® (Bortezomib), Alkeran® (Melphalan) und Prednison geeignet. Sie hat aber etwas mehr Komplikationen zu Folge als bei jüngeren Patienten, und muss daher ärztlich gut überwacht werden, kann aber ambulant erfolgen. Revlimid® (Lenalidomid) und Velcade® werden auch im Alter relativ gut vertragen und sind daher auch geeignet für die Therapie dieser Patientengruppe. Eine autologe Stammzelltransplantation verbietet sich aber in der Alterskategorie dieses Patienten weiterhin.

\section{Frage 5}

Antwort d) ist richtig. 
Das MGUS ist zwar prinzipiell nicht behandlungsbedürftig. Dies kann sich aber ändern, wenn eine Polyneutropathie auftritt, die mit Wahrscheinlichkeit auf das Paraprotein zurückgeführt werden muss.

Patienten mit einem MGUS müssen jährlich ärztlich nachkontrolliert werden, um den Übergang in ein Multiples Mmyelom rechtzeitig zu erkennen und damit schwerwiegende Komplikationen zu verhindern. Auch wenn fast jedem Multiplen Myelom ein MGUS vorausgeht, so entwickelt sich bei weitem nicht aus jedem MGUS ein Multiples Myelom. Die Rate ist durchschnittlich nur 1\% pro Jahr (und nicht 10\%/Jahr); nur in der Hoch-Risiko Gruppe werden mehr als die Hälfte der Patienten in zehn Jahren ein Multiples Myelom entwickeln. 